\title{
THE STABILITY OF TRIPLE STELLAR SYSTEMS
}

\author{
T. A. AGEKJAN and J. P. ANOSOVA \\ Astronomical Observatory, University of Leningrad, Leningrad, U.S.S.R.
}

The motion of triple stellar systems whose components are at rest at the initial epoch is studied. In this study all the components have equal masses, and at the initial epoch the component $A$ is at $\left(-\frac{1}{2}, 0\right)$ on the $(x, y)$-plane, $B$ is at $\left(\frac{1}{2}, 0\right)$, and $C$ is anywhere in the region $(s)$ bounded by the positive $x$-axis, $y$-axis and a line expressed by $\left(x+\frac{1}{2}\right)^{2}+$ $+y^{2}=1$. By this way all the possible initial configurations are given.

If the distance between any two components exceeds $D$, the largest possible value of the smallest distance, the system is called in the state of ejection at this epoch. There are two kinds of ejections, one with escape (disintegration) and one with return. It can be shown that the ejection starts when the distance of at least one component to the center of masses exceeds $3^{1 / 2} G /(-E)$, where $E$ and $G$ are, respectively, the energy of the system and the gravitation constant.

To each initial configuration corresponds the time of disintegration when the ejection with escape starts and the number of returns for the ejection with return.

The region $(s)$ is divided into smaller regions $\left(s^{\prime}\right)$ by lines of instability. When the initial position of $C$ crosses one of this lines the number $n$ changes. The value of the change is not restricted. On the other hand the time of disintegration is a continuous function of the initial coordinates of $C$ within the region $\left(s^{\prime}\right)$ and is infinity on the line of instability.

The regions $\left(s^{\prime}\right)$ are divided into more smaller regions $\left(s^{\prime \prime}\right)$ by lines of the change of the number $n$ to one unity. When the initial position of $C$ crosses one of this lines the number $n$ becomes greater or smaller to one unity, the time of disintegration remains continuous. Within the region ( $\left.s^{\prime \prime}\right)$ the number $n$ is constant.

The area of regions $\left(s^{\prime}\right)$ and $\left(s^{\prime \prime}\right)$ are approximately proportional to $n^{-2}$. 